\title{
Pulse Contour Methods to Estimate Cardiovascular Indices in Micro- And Hypergravity
}

\author{
Tatsuya Arai, Ulrich Limper, Peter Gauger, \\ AND LUIS BECK
}

\begin{abstract}
Arai T, Limper U, Gauger P, Beck L. Pulse contour methods to estimate cardiovascular indices in micro- and hypergravity. Aviat Space Environ Med 2013; 84:1178-85.

Background: The importance of noninvasive health monitoring in space increased as a result of the long-duration missions on the International Space Station (ISS). In order to monitor changes in cardiovascular indices such as cardiac output (CO) and total peripheral resistance (TPR), many methods have been developed using signal processing and mathematical modeling techniques. However, their performance in various gravitational conditions has not been known. Methods: The present study compared 10 methods to estimate $\mathrm{CO}$ and TPR by processing peripheral arterial blood pressure signals recorded from 8 subjects in multiple gravity levels $(1 \mathrm{G}, 0 \mathrm{G}$, and $1.8 \mathrm{G}$ ) during parabolic flights. For reference data sets, CO and TPR were simultaneously obtained by an inert gas rebreathing technique. Root normalized mean square errors and Bland-Altman plots were used to evaluate the estimation methods. Results: The corrected impedance method achieved the lowest estimation errors (20.0\% CO error and $23.5 \%$ TPR error) over the three gravity levels. In microgravity, mean arterial pressure was also demonstrated to be an indicator of CO (24.5\% error). Discussion: The corrected impedance method achieved low estimation errors for a wide range of the gravity levels. Gravity-dependent performance was observed in the mean arterial pressure method that achieved low errors in the shortterm 0 G.

Keywords: inert gas rebreathing, parabolic flight, cardiac output, total peripheral resistance.
\end{abstract}

$\mathrm{T}$ HE IMPACT OF SHORT and long-lasting weightlessness on the cardiovascular system, especially presyncope and fainting of astronauts after spaceflight missions (postflight orthostatic hypotension or orthostatic intolerance), has been in the focus of cardiovascular research for decades. It has been found that postflight orthostatic intolerance is caused by changes in cardiac output (CO) and vasoconstriction deficiencies before and after spaceflight $(17,19)$. As human spaceflight entered the era of long-duration missions on the International Space Station (ISS), the importance of noninvasive health monitoring has increased in order to track changes of the cardiovascular indices and find any sign of deconditioning during and after spaceflight.

The noninvasive cardiovascular monitoring in spaceflight includes infrared finger photoplethysmography (IFP) during former Space Shuttle and Russian MIR space station missions as well as ISS missions $(3,4,10)$. The recorded peripheral arterial blood pressure (ABP) signal can be processed to track changes in $\mathrm{CO}$ and total peripheral resistance (TPR). So-called pulse contour methods (PCMs) have been studied by many researchers $(9,14,25)$ to track changes in CO and TPR, and have been introduced into clinical medicine and physiological research $(3,4,21)$. However, just a few methods are currently used in clinical medicine on a regular basis (22). For example, the corrected aortic input impedance method introduced by Wesseling et al. is used for stroke volume (SV) estimation by pulse wave analysis (3). However, ground-based artificial gravity studies have shown that the model flow method, which was developed from the corrected impedance method, achieves different reliabilities for measurements in different body positions (6). Furthermore, the impact of microgravity on the performance of PCMs is not known. The purpose of this study was, therefore, to compare PCMs using noninvasive beat-to-beat finger blood pressure in various gravity conditions.

\section{METHODS}

\section{Equipment}

Cardiovascular data sets of noninvasive ABP, heart rate (HR) by electrocardiography, and $\mathrm{CO}$ were simultaneously collected during the parabolic flight campaigns of the German Aerospace Center (DLR) in 2010-2012 in Bordeaux, France, and the French Space Agency Centre National d'Etudes Spatiales during the 2011 Paris Le Bourget Air Show. All flights were performed by the NOVESPACE Company using an Airbus A300. The Finometer MIDI ${ }^{\circledR}$ system (Finapres Medical Systems, Amsterdam, The Netherlands) for blood pressure measurement, the Innocor ${ }^{\circledR}$ rebreathing system (Innovision A/S, Odense, Denmark) for obtaining cardiac output $\left(\mathrm{CO}_{\mathrm{REB}}\right)$, and the Biopac ${ }^{\circledR}$ system (Biopac Systems, Goleta, CA) for data acquisition were used. The raw data of $A B P$ and $H R$ were stored via $A C Q^{\circledR}$ software

From the Massachusetts Institute of Technology, Cambridge, MA; the DLR Institute of Aerospace Medicine, Space Physiology Branch, Cologne, Germany; and the University of Witten/Herdecke, Department of Anesthesiology and Intensive Care Medicine, Merheim Medical Center, Hospitals of Cologne, Cologne, Germany.

This manuscript was received for review in March 2013. It was accepted for publication in June 2013.

Address correspondence and reprint requests to: Tatsuya Arai, 77 Massachusetts Ave., Cambridge, MA 02139; tatsuya@alum.mit.edu.

Reprint \& Copyright (C) by the Aerospace Medical Association, Alexandria, VA.

DOI: $10.3357 /$ ASEM.3683.2013 
(Biopac Systems). The details of the in-flight experimental setup has been described previously (15).

\section{Subjects}

In short, $\mathrm{ABP}$ and $\mathrm{HR}$ data were continuously recorded throughout the flights at a $200 \mathrm{~Hz}$ sampling rate from eight healthy subjects in the standing position (four women, $30 \pm 3 \mathrm{yr}, 167 \pm 7 \mathrm{~cm}, 61 \pm 18 \mathrm{~kg}$; and four men, $35 \pm 9 \mathrm{yr}, 173 \pm 9 \mathrm{~cm}, 66 \pm 10 \mathrm{~kg}$ ). All the test subjects had experienced parabolic flights previously. They received scopolamine-hydrobromide injected subcutaneously as a countermeasure for motion sickness before the flights [175 $\mu \mathrm{g}$ (men); $125 \mu \mathrm{g}$ (women)]. The study protocols had been approved in advance by the pertinent French authorities (Agence française de securité sanitaire des produits de sanité). Each subject provided written informed consent before participation.

\section{Procedure}

The following PCMs were applied to the aforementioned parabolic flight ABP data.

Mean arterial pressure: This method is one of the most simple estimators. $\mathrm{CO}$ is proportional to mean arterial pressure (MAP):

$$
C O \propto M A P=\frac{1}{T} \int P(t) d t
$$

where $\mathrm{T}$ is the beat duration and $\mathrm{P}(\mathrm{t})$ is $\mathrm{ABP}$.

Pulse pressure (PP) (7):

$$
C O \propto \sum_{i} \frac{P P_{i}}{T_{i}}=\sum_{i} \frac{S B P_{i}-D B P_{i}}{T_{i}}
$$

where $i$ represents the beat number, and $\mathrm{SBP}_{\mathrm{i}}$ and $\mathrm{DBP}_{\mathrm{i}}$ represent systolic and diastolic blood pressure values of the beat, respectively. Taking DBP into consideration, this method regards the difference of SBP and DBP as an indicator of CO. The PP method is simple and one of the most intuitive and arguably most commonly used in clinical settings (5).

Herd's pulse pressure (PP2) (9):

$$
C O \propto \sum_{i} \frac{M A P_{i}-D B P_{i}}{T_{i}}
$$

In the aforementioned PP method, SBP was replaced by MAP, which is known to be robust against pressure waveform distortion caused by the tapered and bifurcated arterial tree structure.

Modified Herd's method (2):

$$
C O \propto \sum_{i} \frac{\int_{\text {Systole }} P_{i}(t) d t-D B P_{i}}{T_{i}}
$$

This method uses only the systolic ABP signal in the Herd's method.

Liljestrand-Zander's method (14):

$$
C O \propto \sum_{i} \frac{S B P_{i}-D B P_{i}}{S B P_{i}+D B P_{i}} / T_{i}
$$

This method adds a scaling factor of $1 /(\mathrm{SBP}+\mathrm{DBP})$ to the PP method.

Area under the systolic pressure curve:

$$
C O \propto \sum_{i} \frac{\int_{\text {systole }} P_{i}(t) d t}{T_{i}}
$$

Rather than taking the average of an entire single beat, this method uses only the systolic pressure signal to obtain proportional CO. This method overcomes the disadvantage of the MAP method's tendency to underestimate CO when diastolic period is abnormally extended, regardless of the systolic period.

Area under the systolic pressure curve above DBP:

$$
C O \propto \sum_{i} \frac{\int_{\text {systole }}\left(P_{i}(t)-D B P_{i}\right) d t}{T_{i}}
$$

Only the ABP signal above DBP is considered.

Corrected impedance (25):

$$
C O \propto \sum_{i} \frac{\left(163+H R_{i}-0.48 \cdot M A P_{i}\right) \int_{t^{E D}{ }_{i}}^{t^{E S}}\left(P_{i}(t)-D B P_{i}\right) d t}{T_{i}}
$$

where $\mathrm{t}^{\mathrm{ED}}{ }_{\mathrm{i}}$ and $\mathrm{t}^{\mathrm{ES}}{ }_{\mathrm{i}}$ represent time stamps at the end diastole and end systole of the $i_{\text {th }}$ beat, respectively.

Kouchoukos correction (13):

$$
C O \propto \sum_{i} \frac{\left(1+\frac{T_{i}^{S}}{T_{i}^{D}}\right)_{t^{E D_{i}}}^{t_{i}^{E S}} \int_{i}^{i}\left(P_{i}(t)-D B P_{i}\right) d t}{T_{i}}
$$

where $\mathrm{T}^{\mathrm{S}}$ and $\mathrm{T}^{\mathrm{D}}$ represent systolic and diastolic durations, respectively.

$A C$ power: Adopted by the commercial LiDCO Plus PulseCO method of pulse power analysis (20; LiDCO Ltd., London, UK), this makes use of the power of the ABP signal. The beat power factor is proportional to the nominal $\mathrm{CO}$ ejected into the aorta.

$$
C O \propto \frac{\sqrt{\frac{1}{T} \int_{T}(P(t)-M A P)^{2} d t}}{T}
$$

Some of the CO estimation methods require identification of an end systolic time stamp. In this section, methods to identify the end systole from the ABP signal are introduced.

Exponential model: Weissler et al. (24) obtained the relationship between systolic duration and preceding RR interval, describing it as a saturating exponential curve. For the present study, the following equation was adopted to identify the systolic duration of the current beat (2):

$$
\text { Sys }_{i}=436\left(1-\exp \left(-0.0057 R R_{i-1}^{\text {measured }}\right)\right)
$$

Partial pulse pressure model (1): An end diastole always comes after a systolic peak. At the end systole, the pressure value is equal to or lower than SBP. When the pressure drops from SBP to $\alpha \% \mathrm{PP}$ at the end systole, the end 
TABLE I. CARDIOVASCULAR DATA OBTAINED DURING CO REBREATHING MEASUREMENTS IN $\mathbf{N}=\mathbf{8}$.

\begin{tabular}{|c|c|c|c|c|c|c|c|c|c|c|c|c|c|c|c|c|}
\hline \multirow[b]{2}{*}{ Subjects } & \multicolumn{4}{|c|}{$\mathrm{CO}_{\mathrm{REB}}\left(\mathrm{L} \cdot \min ^{-1}\right)$} & \multicolumn{4}{|c|}{ ABP (mmHg) } & \multicolumn{4}{|c|}{ HR (bpm) } & \multicolumn{4}{|c|}{ TPR $[\mathrm{mmHg} /(\mathrm{L} / \mathrm{min})]$} \\
\hline & Max & Min & Mean & SD & Max & Min & Mean & SD & Max & Min & Mean & SD & Max & Min & Mean & SD \\
\hline AA & 9.94 & 4.29 & 7.47 & 2.44 & 143 & 63 & 87.8 & 13.3 & 104 & 34 & 67.6 & 20.4 & 21 & 9 & 12.6 & 3.3 \\
\hline $\mathrm{AO}$ & 5.90 & 2.40 & 4.37 & 1.40 & 136 & 29 & 71.7 & 16.8 & 105 & 57 & 85.6 & 11.1 & 39 & 10 & 18.3 & 7.7 \\
\hline $\mathrm{AM}$ & 8.55 & 3.06 & 5.27 & 2.35 & 205 & 45 & 94.2 & 25.0 & 180 & 25 & 100.0 & 32.7 & 35 & 12 & 20.9 & 7.2 \\
\hline $\mathrm{BX}$ & 14.90 & 4.20 & 7.95 & 3.94 & 154 & 41 & 78.3 & 17.7 & 180 & 47 & 109.6 & 22.2 & 18 & 6 & 11.4 & 3.9 \\
\hline $\mathrm{CC}$ & 11.41 & 5.06 & 7.10 & 1.76 & 176 & 60 & 92.1 & 17.5 & 180 & 64 & 120.5 & 27.7 & 21 & 7 & 13.8 & 4.6 \\
\hline AD & 14.29 & 4.59 & 7.30 & 4.29 & 182 & 45 & 81.7 & 19.0 & 160 & 53 & 108.7 & 26.7 & 18 & 6 & 12.5 & 3.6 \\
\hline$A X$ & 11.34 & 4.66 & 7.42 & 2.04 & 212 & 53 & 104.2 & 25.5 & 180 & 25 & 122.7 & 19.0 & 23 & 9 & 15.1 & 4.6 \\
\hline BR & 12.29 & 3.64 & 7.18 & 2.62 & 183 & 46 & 94.7 & 20.8 & 128 & 62 & 91.2 & 12.7 & 25 & 7 & 14.9 & 5.7 \\
\hline
\end{tabular}

systolic pressure can be described as $\mathrm{P}_{\mathrm{ES}}=\mathrm{P}_{\mathrm{D}}+\alpha$ $\left(P_{S}-P_{D}\right)$, where $P_{E S}$ is the time stamp of the estimated end systole. The value of $\alpha$ was changed from 10 to $100 \%$ $\mathrm{PP}$ and applied to the $\mathrm{CO}$ estimation methods that require end systole identifier.

\section{Statistical Analysis}

We applied the aforementioned 10 PCMs to the parabolic flight $\mathrm{ABP}$ data after the experiments using MATLAB $^{\circledR}$ (MathWorks, Natick, MA). The ABP signal during each $\mathrm{CO}$ rebreathing measurement (10 s) was processed. First, peaks in the ABP signal and their preceding local minima were determined as SBP and DBP. The signal between DBPs defines a single beat. The algorithms were applied to calculate SV on a beat-bybeat basis. Then the total SV over time was calculated as a proportional $\mathrm{CO}$ estimate.

Note that all the PCMs provide proportional CO; i.e., the outputs are estimates within a scaling factor. Assuming arterial compliance does not change during testing, in each subject, the proportional CO estimates were scaled as

$$
C_{a}=\frac{\text { mean }(\text { meas })}{\text { mean }(\text { estimated })}
$$

The scaling factor was set to be consistent within each subject (1).

Using the estimated $\mathrm{CO}$ and measured MAP, TPR was also estimated by Ohm's Law: TPR $=$ MAP/CO. The estimated $\mathrm{CO}$ and TPR values were evaluated by comparing with the corresponding reference $\mathrm{CO}\left(\mathrm{CO}_{\mathrm{REB}}\right)$ obtained by Innocor ${ }^{\circledR}$. The reference TPR $\left(\mathrm{TPR}_{\mathrm{REB}}\right)$ was also calculated by Ohm's Law: $\mathrm{TPR}_{\mathrm{REB}}=\mathrm{MAP} / \mathrm{CO}_{\mathrm{REB}}$. As an error criterion, the root normalized mean square error (RNMSE) was adopted:

$$
R N M S E=100 \sqrt{\frac{\sum_{n=1}^{N}\left(\frac{\text { Meas }-E s t}{\text { Meas }}\right)^{2}}{N-N_{f}}}
$$

where Meas, Est, $\mathrm{N}$, and $\mathrm{N}_{\mathrm{f}}$ represent a measured value, estimated value, number of sample, and number of freedom $\left(\mathrm{N}_{\mathrm{f}}=1\right.$, arterial compliance), respectively. The method that achieved the lowest RNMSE was compared with the other methods using the F-test. The statistical significance was defined as $P<0.05$.

\section{RESULTS}

Table I shows an overview of the $\mathrm{CO}_{\mathrm{REB}}, \mathrm{ABP}, \mathrm{HR}$, and TPR characteristics of the eight subjects analyzed. Large interindividual differences are obvious, especially in the ranges of $\mathrm{CO}_{\mathrm{REB}}$, which are larger than those typically seen in hospitalized patients. We analyzed $139 \mathrm{CO}$ data sets, of which 52 sets were collected within $0 \mathrm{G}$, 28 sets in $1.8 \mathrm{G}$, and 59 sets in $1 \mathrm{G}$. The overall results are summarized in Table II, showing that most of the methods generated their largest errors in short-term $0 \mathrm{G}$ and the smallest in short-term $1.8 \mathrm{G}$.

Fig. 1A summarizes the $\mathrm{CO}$ errors by different methods under all tested gravity loads. For methods using

\begin{tabular}{|c|c|c|c|c|c|c|c|c|}
\hline \multirow[b]{2}{*}{ Methods } & \multicolumn{4}{|c|}{ CO RNMSEs } & \multicolumn{4}{|c|}{ TPR RNMSEs } \\
\hline & All Gs & $1 \mathrm{G}$ & $0 \mathrm{G}$ & $1.8 \mathrm{G}$ & All Gs & $1 \mathrm{G}$ & $0 \mathrm{G}$ & $1.8 \mathrm{G}$ \\
\hline Wesseling's corrected impedance (25) & 20.0 & 19.2 & 27.3 & 16.3 & 23.5 & 17.8 & 30.9 & 17.3 \\
\hline Kouchoukos (13) & 21.9 & 19.4 & 29.2 & 18.2 & 31.0 & 18.7 & 41.8 & 28.1 \\
\hline Area under the curve, above DBP & 22.3 & 20.3 & 26.0 & 18.7 & 27.4 & 19.0 & 35.1 & 26.4 \\
\hline Modified Herd's method (2) & 22.4 & 21.0 & 26.8 & 18.8 & 26.4 & 19.6 & 34.7 & 20.0 \\
\hline Area under the curve & 24.7 & 21.5 & 30.3 & 26.1 & 24.3 & 20.8 & 27.7 & 24.4 \\
\hline Herd's pulse pressure (9) & 25.0 & 24.4 & 29.1 & 24.9 & 31.4 & 22.6 & 41.4 & 25.4 \\
\hline AC power (20) & 25.7 & 22.9 & 27.8 & 16.7 & 28.3 & 21.4 & 38.3 & 16.9 \\
\hline Pulse pressure & 28.7 & 25.6 & 29.4 & 35.1 & 33.0 & 23.1 & 45.9 & 19.1 \\
\hline Liljestrand-Zander's method (14) & 29.2 & 25.3 & 25.5 & 18.1 & 30.8 & 24.5 & 40.4 & 20.3 \\
\hline MAP & 33.3 & 26.5 & 24.5 & 28.6 & 34.9 & 24.8 & 44.4 & 32.6 \\
\hline
\end{tabular}

TABLE II. RNMSES OF CO AND TPR ESTIMATION (\%). 
end systolic time stamps, the end systolic identifier that provided the lowest RNMSEs is shown. Among the methods, corrected impedance with $60 \%$ PP as end systole achieved the lowest CO RNMSE (20.0\%). The 1-G loading scenario (Fig. 1B) yielded similar results. Fig. 1C shows the RNMSEs during $0 \mathrm{G}$. The MAP method achieved the lowest RNMSE of $24.5 \%$. Fig. 1D shows the RNMSEs during 1.8 G. The corrected impedance method achieved the lowest RNMSE of $16.3 \%$.

The CO estimation history of the corrected impedance method compared to $\mathrm{CO}_{\mathrm{REB}}$ is shown in Fig. 2A, demonstrating the traceability of changes of $\mathrm{CO}$ by the corrected impedance method over the wide range of measured CO and gravity levels. Kouchoukos (60\% PP as end systole identifier), area under the curve above
DBP (60\% PP as end systole identifier), and modified Herd's (using an exponential model) achieved 21.9\%, $22.3 \%$, and $22.4 \%$ RNMSEs, respectively. Other methods, especially the methods that do not use end systolic time stamps, were found to be statistically larger in RNMSEs (Fig. 1), including the Liljestrand-Zander method (29.2\%, CO estimation history shown in Fig. 2B).

As for TPR estimation, the corrected impedance method achieved 23.5\% RNMSE (Fig. 3A), while the Liljestrand-Zander method achieved 30.8\% RNMSE (Fig. 3B). Bland-Altman plots of the Liljestrand-Zander and the corrected impedance methods are shown in Fig. 4 . The corrected impedance method showed smaller correlation coefficient and $95 \%$ limits of agreement $\left[\mathrm{R}^{2}=0.003\right.$ and $\pm 2.73 \mathrm{mmHg} /(\mathrm{L} / \mathrm{min})$, respectively,

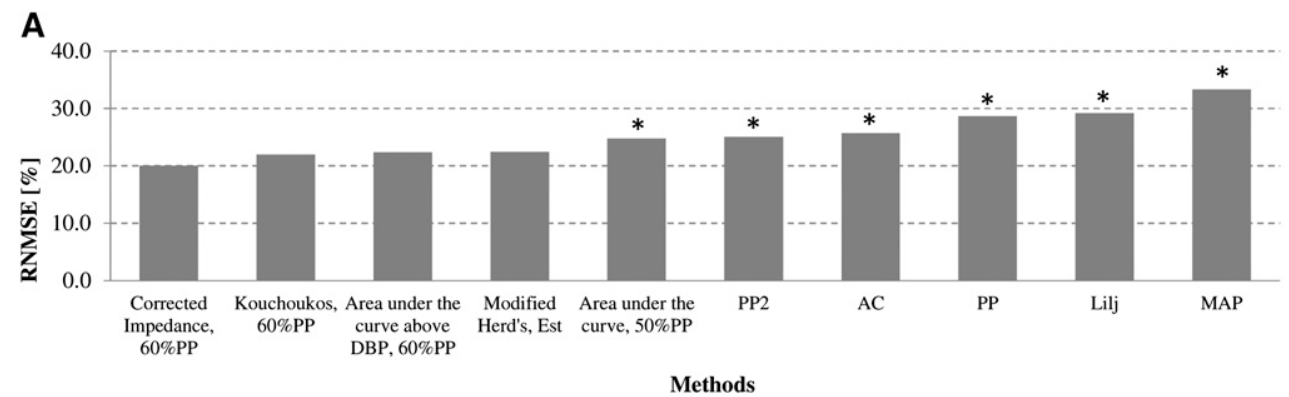

B

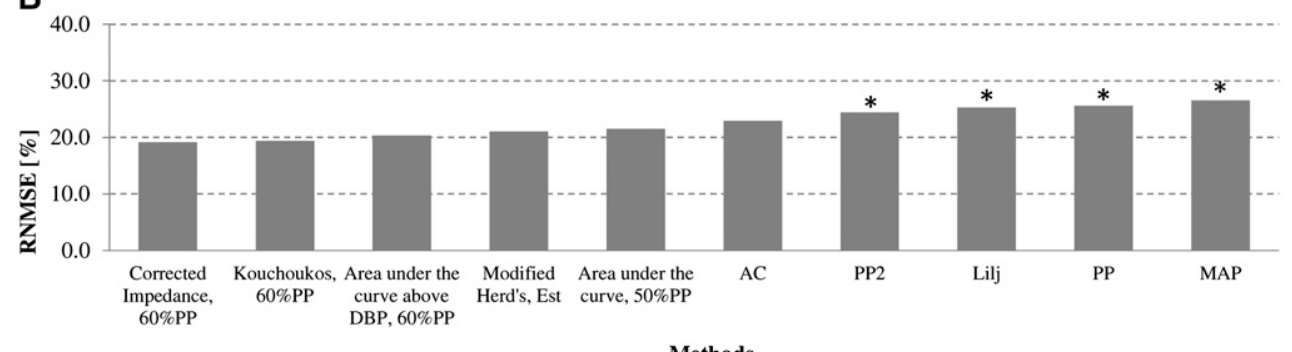

C

Methods

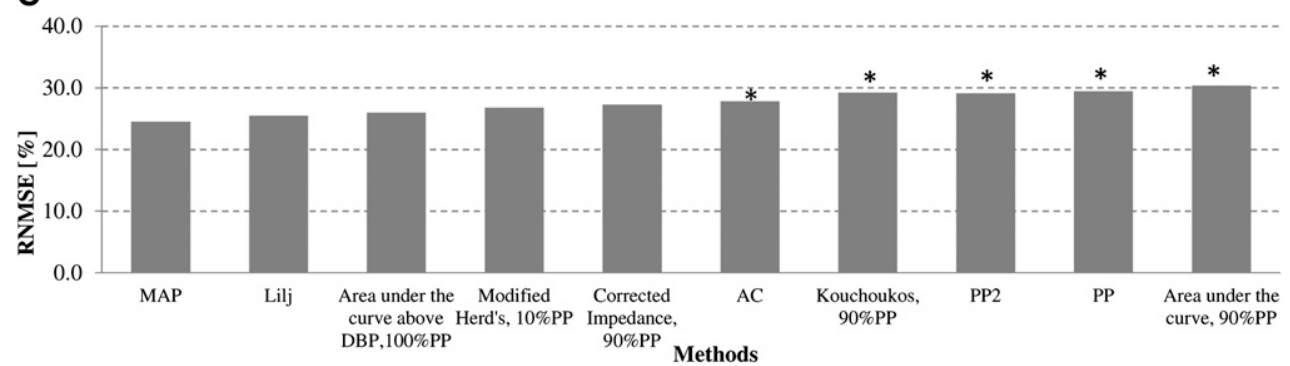

D

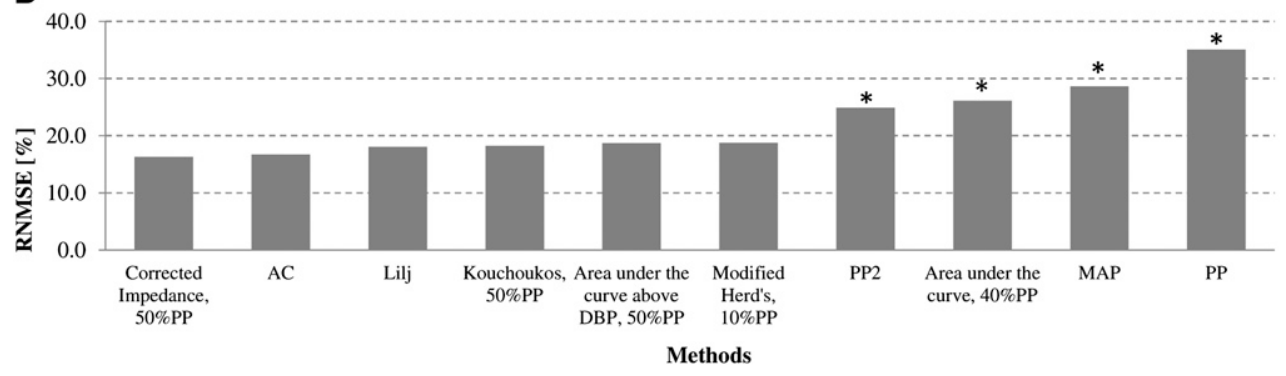

Fig. 1. A) RNMSEs of the CO estimation methods for all the gravity levels. B) RNMSEs of the CO estimation methods in 1 G. C) RNMSEs of the CO estimation methods in $0 \mathrm{G}$. D) RNMSEs of the CO estimation methods in $1.8 \mathrm{G}$. Asterisks represent significant statistical differences $(P<0.05)$ regarding the best method under the particular gravity load. 

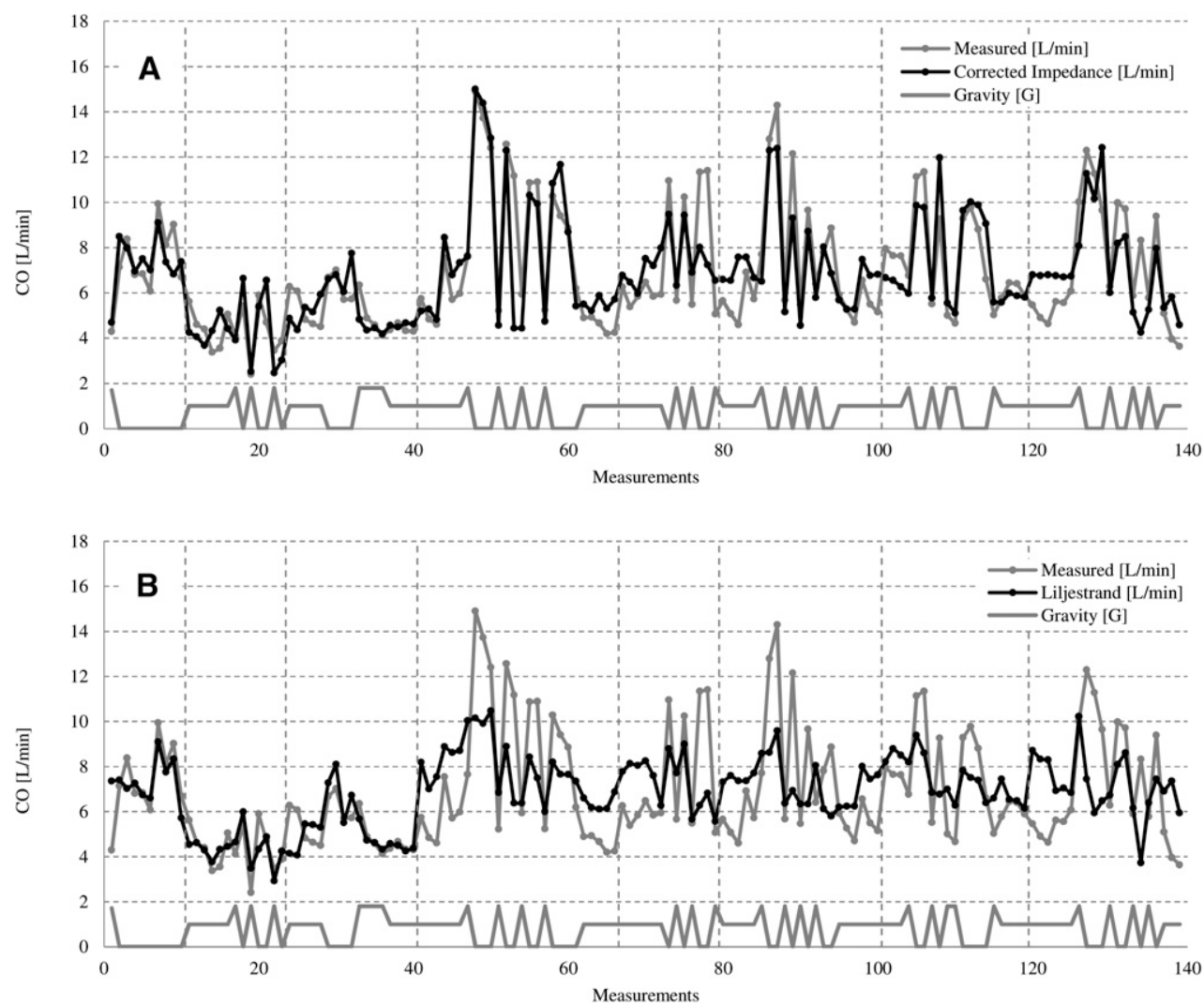

Fig. 2. A) CO estimation history by the corrected impedance method using $60 \%$ PP as end systole. B) CO estimation history by the LiljestrandZander method.

shown in Fig. 4B] than those of the Liljestrand-Zander method $\left[R^{2}=0.020\right.$ and $\pm 4.12 \mathrm{mmHg} /(\mathrm{L} / \mathrm{min})$, respectively, shown in Fig. 4A], indicating independency from the physiological range of true $\mathrm{CO}$ values. Similar trends can be seen in those of TPR estimation (Figs. 4C and D).

\section{DISCUSSION}

Finger blood pressure waves obtained during parabolic flights within wide physiological ranges were processed using $\mathrm{CO}$ estimation methods and including different methods of end systole identification. There were 10 such estimation methods assessed against cardiac output determined by foreign gas rebreathing. The corrected impedance, Kouchoukos, area under the curve (above DBP), and the modified Herd's methods achieved the lowest RNMSEs among all 10 analyzed methods.

The high RNMSEs of the PP method could be attributed to their sensitivity to ABP waveform distortion; SBP tends to increase as the pulse wave travels through the tapered and bifurcated arterial tree. In the meantime, DBP and MAP are known to be robust against distortion. For example, the corrected impedance method and Kouchoukos correction method are based on the area under the curve method, but adopted distortionrobust scaling factors to achieve high estimation accuracy. The corrected impedance method adopted HR and MAP as scaling factors, while the Kouchoukos correction method used the ratio of systolic and diastolic durations.
The error of the MAP method stems from the assumptions of constant TPR. Interestingly, however, the MAP method achieved low error in $0 \mathrm{G}$ (Fig. 1B) with respect to the other methods, which could be attributed to the baroreceptor response induced by the headwards fluid shift; likely the sympathetic system was inhibited excessively, resulting in the saturated and constant vascular response.

Herd's pulse pressure method (PP2) overcame the shortcoming of the PP method by using MAP and DBP, which are both robust against waveform distortion. However, the method is sensitive to variability in the diastolic periods. For example, if SV values are constant for several beats while diastolic periods start to decrease, MAP would decrease and SV values would be underestimated. In that light, the modified Herd's method was developed to overcome the weakness of Herd's pulse pressure method. This method takes into account only the systolic period. Since any abnormally shortened or prolonged diastolic periods (that do not contribute to SV or CO) are excluded, better estimation accuracy was expected. It was also found that the modified Herd's method is relatively insensitive to end systole identification methods (the exponential model or partial PP methods) (2).

Although the Liljestrand-Zander method had been reported by Sun et al. to work best in the data sets of patients on intensive care units (ICU) (21), the method did not achieve low RNMSEs in the present study, especially 

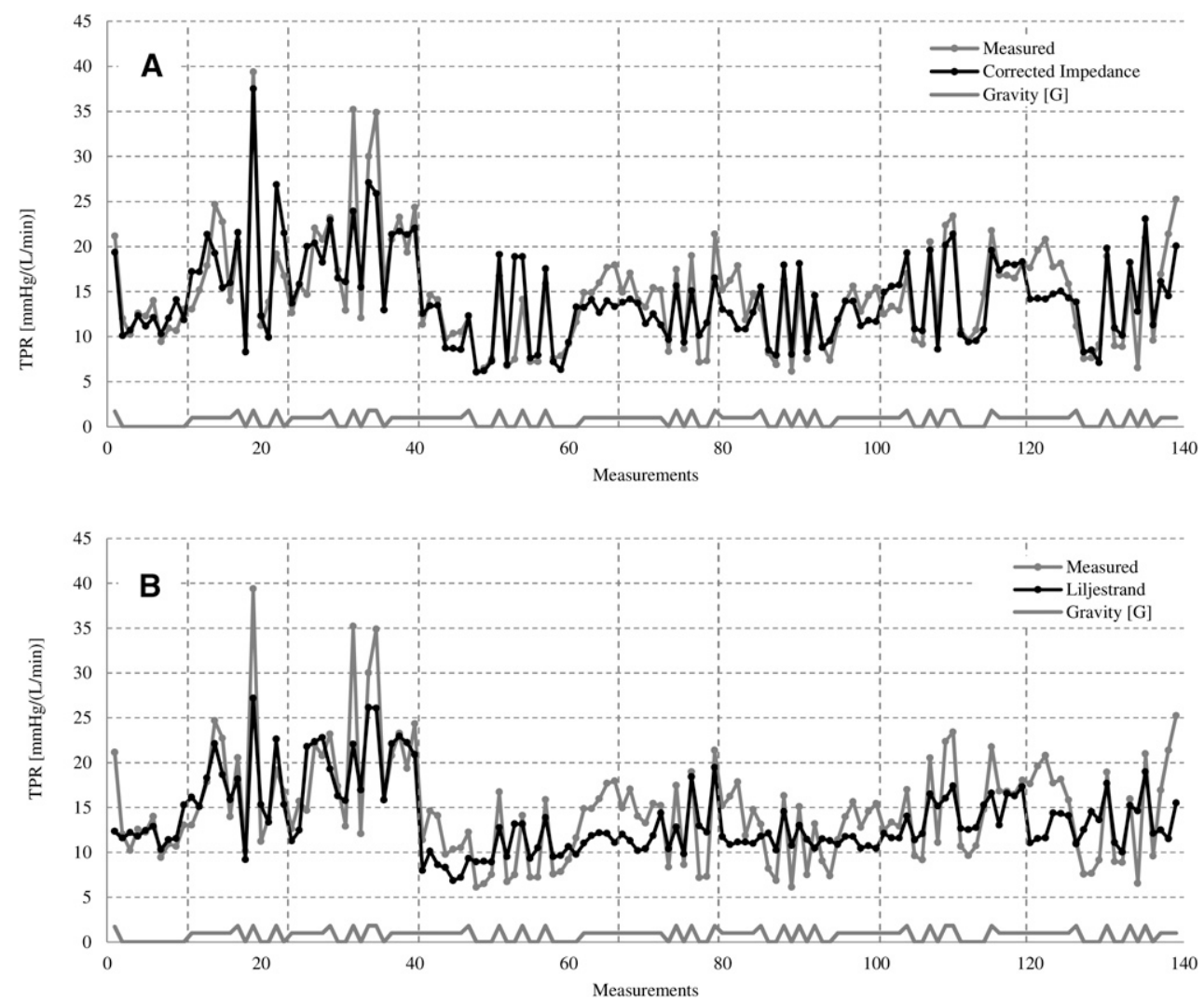

Fig. 3. A) TPR estimation history by the corrected impedance method. B) TPR estimation history by the Liljestrand-Zander method.

in $1 \mathrm{G}$ and $1.8 \mathrm{G}$. The reason could be attributed to stable ICU patient data sets and the averaging effect of the method; the Liljestrand-Zander method normalizes PP by a sum of SBP and DBP. Therefore, it tends to provide relatively flat and stable $\mathrm{CO}$ estimates. In the ICU setting, cardiovascular indices, such as $\mathrm{CO}$ and $\mathrm{ABP}$, of patients are carefully maintained. Thus, the averaging effect of Liljestrand-Zander favorably resulted in low RNMSEs. On the other hand, a wide range of $\mathrm{CO}$ and $\mathrm{ABP}$, as can be seen in the present study, would challenge the method. The German physiologist Gauer pointed out that that this relationship leads to good results if changes in $\mathrm{CO}$ are mainly the result of changes in HR (8). The Liljestrand-Zander formula was often criticized for not taking changes of elasticity and other parameters into account (8). During a parabolic flight maneuver, not only large HR changes, but also exaggerated changes in blood pressure and TPR can be observed (16).

In $0 \mathrm{G}$, higher PP values ( $90 \%$ or $100 \%$ PP) tended to be selected as the indicator of end systole than in other gravity scenarios. These PP values correspond to the beginning of the ABP descent immediately after SBP. This could be explained by early aortic valve closures and shortened systolic phases in the cardiac cycle in $0 \mathrm{G}$ with respect to $1 \mathrm{G}$ and $1.8 \mathrm{G}$. However, a shortened systolic phase in $0 \mathrm{G}$ would not be well in line with the observations of Johns et al. (11), who found an increased left ventricular ejection time (LVET) in $0 \mathrm{G}$ with respect to $1.8 \mathrm{G}$ using ultrasound measurements. Contradictory to the findings of Johns et al. are the findings of Mukai et al.
(18). They used impedance cardiography to evaluate LVET during gravity transitions in the standing position on parabolic flight. They were not able to find a significant change in LVET during the $0-G$ phase with respect to the initial 1.8-G phase. From our point of view it is not clear yet if higher PP values as an end systole indicator in microgravity are just a methodical adjustment or correlate with a true change in LVET.

In $1.8 \mathrm{G}$, the corrected impedance method achieved the lowest RNMSEs of $16.3 \%$ in CO and $17.3 \%$ in TPR. During the microgravity phases, however, it resulted in high RNMSEs. The higher errors could be attributed to the rapid decrease in TPR; acute changes in TPR are known to occur in weightlessness during spaceflight missions and on parabolic flights (16), and low TPR in 0 $\mathrm{G}$ might have resulted in the low accuracy of the corrected impedance method (6). The other PCMs also resulted in higher RNMSEs in $0 \mathrm{G}$, which could be explained by the decreased performance of PCMs in general during low TPR values (12). PCMs assume constant blood volume in the arterial system, while fluid shift between the venous and arterial systems occurs upon a rapid decrease in TPR. Estimation errors would decrease once the transient fluid exchange between the systems is stabilized in a long-duration space mission. In considering the effect of TPR changes, locations of ABP measurement could also influence the results. A lack of agreement of arm and finger ABP was reported during spaceflight (23). The larger brachial arteries could be less subject to TPR changes than the smaller 


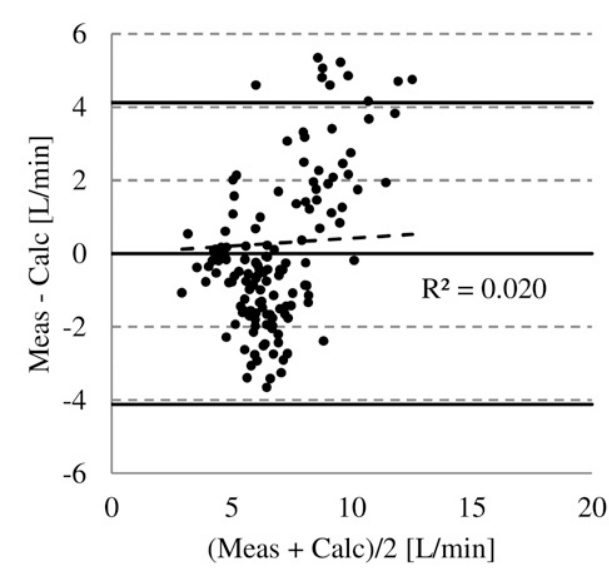

A CO by Liljestrand-Zander

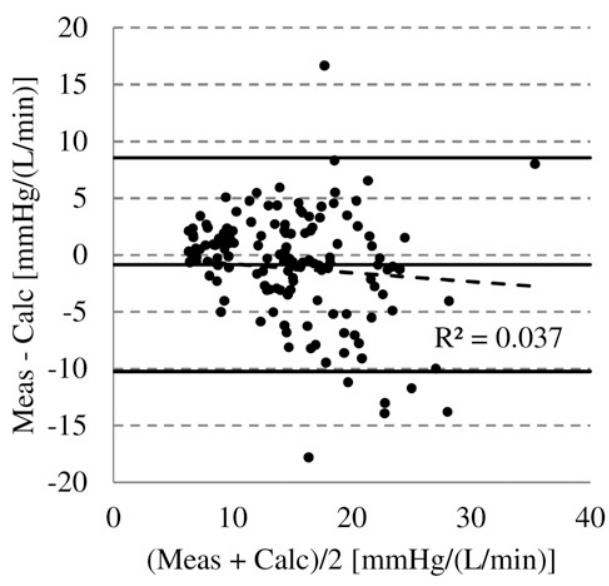

C TPR by Liljestrand-Zander

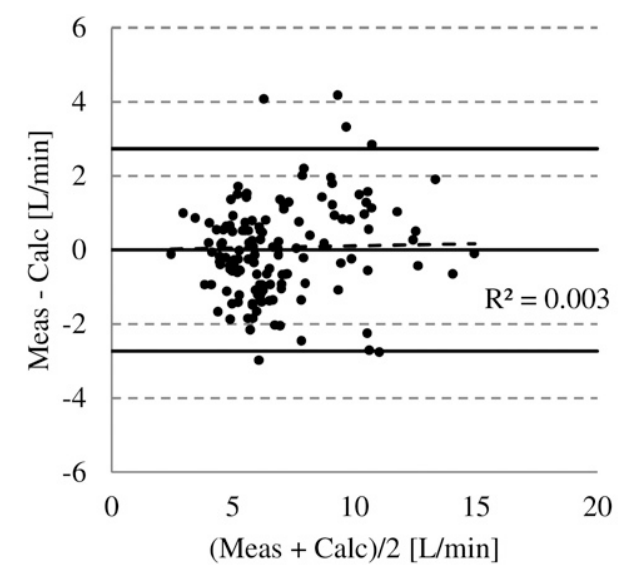

B CO by Corrected Impedance

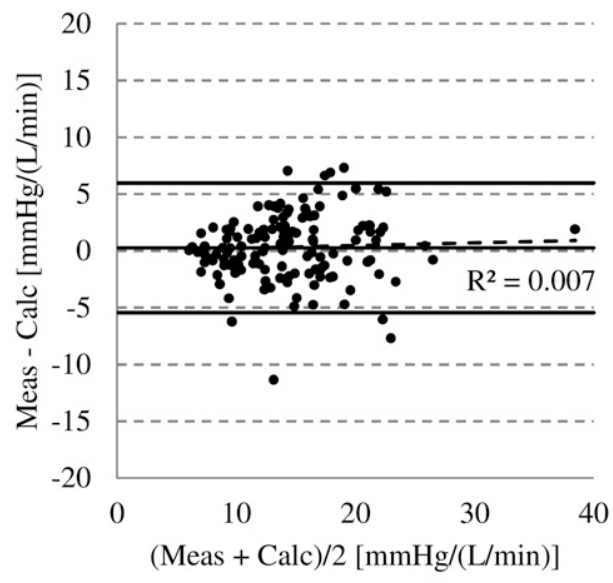

D TPR by Corrected Impedance

Fig. 4. Bland-Altman plots of the Liljestrand-Zander and corrected impedance methods. A) CO by Liljestrand-Zander, $\mathrm{R}^{2}=0.020$. B) CO by corrected impedance, $R^{2}=0.003$. C) TPR by Liljestrand-Zander, $R^{2}=0.037$. D) TPR by corrected impedance, $R^{2}=0.007$.

radial arteries. Comparison of PCMs using brachial and radial arteries during parabolic flight or spaceflight would reveal the robustness of each method in response to acute changes in TPR.

To summarize, we report for the first time comparison of 10 different estimation methods of $\mathrm{CO}$ by processing peripheral arterial blood pressure signals under the changing gravity conditions of parabolic flights. Our main findings are: 1) changing performance of each pulse pressure estimation method was observed under changing $G$ loads; 2) the corrected impedance method worked best on average in all tested $G$ loads; 3 ) surprisingly, MAP showed the best performance of all tested methods in short-term $0 \mathrm{G}$; and 4) most of the methods showed the lowest errors in $1.8 \mathrm{G}$ and the highest errors in $0 \mathrm{G}$. These preliminary results suggest that using multiple PCMs, rather than a single PCM, would be advantageous in analyzing cardiovascular systems under varying G-loads. More precisely, using the corrected impedance method for blood pressure data taken in $1 \mathrm{G}$ and short-term $1.8 \mathrm{G}$, and the MAP method for the data in short-term $0 \mathrm{G}$ might decrease the overall error of $\mathrm{CO}$ estimation.

We acknowledge the following limitations of the study: the sample size $(N=8)$ was small due to the restriction imposed by the number of parabolic flight test subjects. The phases of different $G$ loads lasted just few seconds and further studies are necessary to compare the estimation methods during long-duration spaceflight missions. The accuracy of the CO and TPR reference method as well as the noninvasive IFP were limited (15), which might result in the large errors of all the estimation methods $(>20 \%)$ under the changing gravity conditions. Therefore, conclusions from analysis of IFP data should be drawn carefully. The most accurate cardiovascular monitoring techniques would be invasive blood flow and pressure measurement, such as an ultrasound flow probe and a catheter pressure sensor. Nevertheless, inert gas rebreathing and IFP are well-established, safe, and regularly used methods of reference $\mathrm{CO}$ determination and blood pressure measurement on spaceflight missions and parabolic flights. Further tuning of 
the algorithm parameters in using IFP would increase the estimation accuracy. Future work should also include application of the PCM methods to ABP data sets recorded during a long-duration spaceflight on ISS. Due to the stabilized arterial blood volume in $0 \mathrm{G}$, lower estimation errors are expected.

\section{ACKNOWLEDGMENTS}

We are grateful for the test subjects' participation in the parabolic flight experiments. The authors wish to thank the German Aerospace Center (DLR) and the French National Center for Space Studies (CNES) for providing the flight opportunities during the $15^{\text {th }}, 16^{\text {th }}$, and $19^{\text {th }}$ DLR parabolic flight campaigns and the demonstration flight during the 2011 Paris Le Bourget Air Show. We are also thankful to W. Sies for his technical support and to the appointees of NOVESPACE for all their skillful help.

Authors and affiliations: Tatsuya Arai, M.S., Ph.D., Massachusetts Institute of Technology, Cambridge, MA; Ulrich Limper, M.D., Department of Anesthesiology and Operative Intensive Care Medicine, Medical Center Cologne-Merheim, University of Witten/ Herdecke, Cologne, Germany; and Peter Gauger, M.Eng., and Luis Beck, M.D., German Aerospace Center (DLR), Cologne, Germany.

\section{REFERENCES}

1. Arai T. Estimation of cardiovascular indices by analysis of the arterial blood pressure signal [Doctoral Thesis]. Cambridge, MA: Massachusetts Institute of Technology; 2011.

2. Arai T, Lee K, Marini RP, Cohen RJ. Estimation of changes in instantaneous aortic blood flow by the analysis of arterial blood pressure. J Appl Physiol 2012; 112:1832-8.

3. Baisch F, Beck L, Blomqvist G, Wolfram G, Drescher J, et al. Cardiovascular response to lower body negative pressure stimulation before, during, and after space flight. Eur J Clin Invest 2000; 30:1055-65.

4. Charles J. Studies of orthostatic intolerance with the use of lower body negative pressure (LBNP). Mir 18 final science report. Houstson, TX: National Aeronautics and Space Administration, Lyndon B. Johnson Space Center; 1998.

5. Dart AM, Kingwell BA. Pulse pressure-a review of mechanisms and clinical relevance. J Am Coll Cardiol 2001; 37:975-84.

6. Dyson KS, Shoemaker JK, Arbeille P, Hughson RL. Model flow estimates of cardiac output compared with Doppler ultrasound during acute changes in vascular resistance in women. Exp Physiol 2010; 95:561-8

7. Erlanger J, Hooker DR. An experimental study of blood-pressure and of pulse-pressure in man. Baltimore, MD: Johns Hopkins Hospital; 1904.

8. Gauer OH. Blood circulation. In: Gauer OH, Kramer K, Jung R, eds. München-Berlin-Wien, Germany: Urban \& Schwarzenberg; 1972.

9. Herd JA, Leclair NR, Simon W. Arterial pressure pulse contours during hemorrhage in anesthetized dogs. J Appl Physiol 1966; 21:1864-8.

10. Hughson RL, Shoemaker JK, Blaber AP, Arbeille P, Greaves $\mathrm{DK}$, et al. Cardiovascular regulation during long-duration spaceflights to the International Space Station. J Appl Physiol 2012; 112:719-27.

11. Johns JP, Vernalis MN, Karemaker JM, Latham RD. Doppler evaluation of cardiac filling and ejection properties in humans during parabolic flight. J Appl Physiol 1994; 76:2621-6.

12. Junttila EK, Koskenkari JK, Ohtonen PP, Ala-Kokko TI Uncalibrated arterial pressure waveform analysis for cardiac output monitoring is biased by low peripheral resistance in patients with intracranial haemorrhage. Br J Anaesth 2011; 107:581-6.

13. Kouchoukos NT, Sheppard LC, McDonald DA. Estimation of stroke volume in the dog by a pulse contour method. Circ Res 1970; 26:611-23.

14. Liljestrand G, Zander E. Vergleichende Bestimmungen des Minutenvolumens des Herzens beim Menschen mittels der Stickoxydulmethode und durch Blutdruckmessung [Comparison of cardiac output determination by the nitrous oxide method and blood pressure measurement in humans]. Z Gesamte Exp Med 1928; 59:105-22 (in German).

15. Limper U, Gauger P, Beck LE. Upright cardiac output measurements in the transition to weightlessness during parabolic flights. Aviat Space Environ Med 2011; 82:448-54.

16. Liu J, Verheyden B, Beckers F, Aubert AE. Haemodynamic adaptation during sudden gravity transitions. Eur J Appl Physiol 2012; 112:79-89.

17. Meck JV, Waters WW, Ziegler MG, deBlock HF, Mills PJ, et al. Mechanisms of postspaceflight orthostatic hypotension: low alpha1-adrenergic receptor responses before flight and central autonomic dysregulation postflight. Am J Physiol Heart Circ Physiol 2004; 286:H1486-95.

18. Mukai CN, Lathers CM, Charles JB, Bennett BS. Cardiovascular responses to repetitive exposure to hyper- and hypogravity states produced by parabolic flight. J Clin Pharmacol 1994; 34:472-9.

19. Perhonen MA, Franco F, Lane LD, Buckey JC, Blomqvist CG, et al. Cardiac atrophy after bed rest and spaceflight. J Appl Physiol 2001; 91:645-53.

20. Rhodes A, Sunderland R. Arterial pulse power analysis: the $\mathrm{LiDCO}^{\mathrm{TM}}$ plus system. In: Pinsky MR, Payen D, eds. Update in intensive care and emergency medicine. Berlin: SpringerVerlag; 2005:183-92.

21. Sun JX, Reisner AT, Saeed M, Heldt T, Mark RG. The cardiac output from blood pressure algorithms trial. Crit Care Med 2009; 37:72-80.

22. Truijen J, van Lieshout JJ, Wesselink WA, Westerhof BE. Noninvasive continuous hemodynamic monitoring. J Clin Monit Comput 2012; 26:267-78.

23. Verheyden B, Liu J, Beckers F, Aubert AE. Adaptation of heart rate and blood pressure to short and long duration space missions. Respir Physiol Neurobiol 2009; 169(Suppl. 1):S13-6.

24. Weissler AM, Harris LC, White GD. Left ventricular ejection time index in man. J Appl Physiol 1963; 18:919-23.

25. Wesseling KH, Jansen JR, Settels JJ, Schreuder JJ. Computation of aortic flow from pressure in humans using a nonlinear, threeelement model. J Appl Physiol 1993; 74:2566-73. 